Ophthalmologe 2020 $\cdot 117: 403-404$

https://doi.org/10.1007/s00347-020-01066-w

(c) Springer Medizin Verlag GmbH, ein Teil von Springer Nature 2020

Karl Boden ${ }^{1,2}$

${ }^{1}$ Augenklinik Sulzbach, Knappschaftsklinikum Saar, Sulzbach/Saar, Deutschland

${ }^{2}$ Klaus Heimann Eye Research Institute Sulzbach/Saar, Sulzbach/Saar, Deutschland

\title{
Femtosekundenlaser-assistierte Kataraktchirurgie
}

Seit Beginn der Staroperation in der frühen Antike hat sich das Operationsverfahren immer wieder den aktuellen technischen Möglichkeiten angepasst. Innovative Entwicklungen wie Implantation einer künstlichen Hinterkammerlinse durch Sir Harold Ridley oder die kontinuierliche Kapsulorhexis durch Neuhann und Gimbel waren Meilensteine, die uns die heutige Kleinschnitttechnik, die fast 1 Mio. mal im Jahr in Deutschland durchgeführt wird, ermöglicht hat.

Einige technische Verbesserungen waren bei ihrem Start mit einer intensiven Diskussion vergesellschaftet. Ähnlich zu der hitzigen Debatte bei der Einführung der Phakoemulsifikation durch Charles Kelman hat auch die Integration des Femtosekundenlasers in die Kataraktoperation in der Anfangsphase heftige Diskussionen ausgelöst. Zum einen lag dies sicherlich an der stark durch die Industrie geführten Debatte, die einer Marketingkampagne glich, und dass der Disput unter den Ärzten nicht auf medizinisch wissenschaftlicher Grundlage geführt wurde. Zum anderen konnte alleine durch den zeitlichen Aspekt nicht auf eine fundierte Datenlage für die Argumentation zurückgegriffen werden. Da wir jetzt als Operateure auf eine über 10-jährige Erfahrung mit dem Femtosekundenlaser in der Kataraktchirurgie zurückblicken, ist die Sachlage für eine Bewertung der Technologie eine vollkommen andere.

Der Femtosekundenlaser ermöglicht uns, mit einer verbesserten Präzision und Reproduzierbarkeit der Schnittführung neue Wege in der Kataraktchirurgie zu gehen. Die Grundlagen dafür schuf Donna Strickland, Laserphysikerin und Pro- fessorin an der University of Waterloo in Ontario. Sie ist erst die dritte Frau, die dafür 2018 den Nobelpreis für Physik erhielt. Strickland wurde gemeinsam mit ihrem Doktorvater Gérard Mourou für die Entwicklung der „chirped pulse amplification“ (CPA), die Grundlage für den Femtosekundenlaser, ausgezeichnet. Die beiden teilten sich den Preis mit Arthur Ashkin, der ebenfalls wichtige Forschungsarbeit für die Entwicklung der „optischen Pinzette“ geleistet hat.

\section{》) Der Femtosekundenlaser ermöglicht neue Wege in der Kataraktchirurgie}

Die beiden Physiker entwickelten mit CPA eine Methode, um Laserpulse zunächst räumlich zu strecken und sie dann einzeln zu verstärken. So entstehen sehr kurze, sehr energiereiche Pulse. Diese enthalten zwar - jeder Puls für sich sehr hohe Energien, aber weil sie unfassbar kurz sind, führen sie nicht zu einer Zerstörung des gelaserten Objekts. Daher sind die Laser im Fokus extrem präzise als Werkzeug zu nutzen.

Es ist richtig, wenn neue Technologien wie die der CPA in Gestalt des Femtosekundenlasers zunächst kritisch begutachtet werden. Aber man sollte sich als Arzt mit den wissenschaftlichen Erkenntnissen und den Ergebnissen auseinandersetzen und diese in das eigene Arbeiten mit einfließen lassen.

Wir möchten Ihnen deswegen mit diesem Heft zu der Femtosekundenlaserassistierten Kataraktchirurgie den aktuellsten Stand der wissenschaftlichen Erkenntnisse wiedergeben, um deutlich $\mathrm{zu}$ machen, dass diese Technologie ein fes- 
ter Bestandteil für die nächste Entwicklungsstufe zur Kataraktoperation 4.0 ist.

Zunächst werden wir Ihnen einen Überblick über den aktuellen Stellenwert der Femtosekundenlaser-assistierten Kataraktchirurgie (FLACS) geben. Anschließend werden Herr Mirshahi und Frau Latz Ihnen die Möglichkeiten und die Limitationen der Astigmatismuskorrigierenden Keratotomie erläutern. Die Kollegen Holland und Rüfer werden in ihrem Beitrag die klaren Vorteile der speziell für die FLACS designten Intraokularlinsen (IOL) darstellen, die uns neue Möglichkeiten der IOL-Zentrierung und eine klarere Aussage über die postoperative effektive Linsenposition ermöglichen.

Über die Zukunft des Femtosekundenlasers in der Kataraktchirurgie werden Herr Dick und Herr Schulz berichten. Mit ihrer Ausführung wird deutlich, dass sich in unmittelbarer Zukunft sehr viel in der Kataraktchirurgie verändert, was enormen Einfluss auf die tägliche Versorgung unserer Patienten haben wird.

Wir wünschen Ihnen viel Spaß beim Lesen dieser Ausgabe und freuen uns auf eine anregende Diskussion.

Mit freundlichen Grüßen

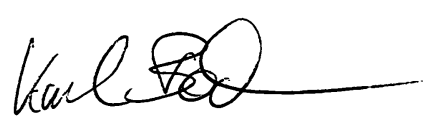

Dr. Karl Boden

\section{Korrespondenzadresse}



Dr. Karl Boden

Augenklinik Sulzbach, Knappschaftsklinikum Saar An der Klinik 10, 66280 Sulzbach/Saar, Deutschland Karl.Boden@kksaar.de

Interessenkonflikt. K. Boden ist Referent für die Firma Ziemer Deutschland GmbH.

In eigener Sache



\section{Auch online Zugang zu allen Beiträgen von Der Ophthalmologe}

Wussten Sie, dass Sie als Abonnent dieser Zeitschrift automatisch online auf das gesamte Beitragsarchiv zugreifen können?

Als Abonnent von Der Ophthalmologe oder Mitglied der Deutschen Ophthalmologischen Gesellschaft (DOG) erhalten Sie zwölf mal im Jahr Ihre Ausgabe nach Hause geliefert.

Damit nicht genug: Im Abonnement ist auBerdem der Zugriff auf das gesamte OnlineArchiv Ihrer Zeitschrift inbegriffen.

\section{Und so einfach geht es:}

Registrieren Sie sich einmal über www.springermedizin.de/register:

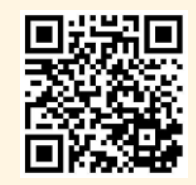

Über diesen QR-Code schnell und einfach registrieren

Bei der Registrierung geben Sie einfach Ihren Vor- und Nachname und Lieferadresse wie beim Abonnement der Zeitschrift (siehe Adressaufkleber auf Ihrem Heft) an. So kann im System die Zugehörigkeit zu Ihrer Zeitschrift sichergestellt werden.
Aufgrund des Heilmittelwerbegesetzes dürfen die Inhalte der Website nur medizinischen Fachkreisen zur Verfügung gestellt werden. Bei der Anmeldung bitten wir Sie deshalb einen Berufsnachweis vorzulegen. Bei Medizinern mit Mitgliedschaft in der deutschen Ärztekammer reicht die einheitliche Fortbildungsnummer (EFN). Alternativ schicken Sie eine Bestätigung des Arbeitgebers, Studiennachweis oder andere Zeugnisse ganz unkompliziert an kundenservice@springermedizin.de.

Mit Benutzername und Passwort haben Sie außerdem Zugang zu den freien Inhalten auf den Seiten von: https://www.springermedizin.de/ https://www.aerztezeitung.de/

Sollten Fragen oder Probleme auftauchen, wenden Sie sich einfach an Ihren Kundenservice:

kundenservice@springermedizin.de 